Original research

\title{
Outcome of a neuromuscular training program on recurrent ankle sprains. Does the initial type of healthcare matter?
}

\author{
Adinda K.E. Mailuhu ${ }^{a, *}$, Marienke van Middelkoop ${ }^{a}$, Sita M.A. Bierma-Zeinstra ${ }^{\text {a }}$, \\ Patrick J.E. Bindels ${ }^{\mathrm{a}}$, Evert A.L.M. Verhagen ${ }^{\mathrm{b}}$ \\ a Department of General Practice, Erasmus MC University Medical Center, Rotterdam, The Netherlands \\ ${ }^{\mathrm{b}}$ Amsterdam Collaboration for Health and Safety in Sports, Department of Public and Occupational Health, Amsterdam Movement Sciences, VU University \\ Medical Center, Amsterdam, The Netherlands
}

\section{A R T I C L E I N F O}

\section{Article history:}

Received 1 October 2019

Received in revised form 9 March 2020

Accepted 16 March 2020

Available online $\mathrm{xxx}$

\section{Keywords:}

Ankle injury

medical consumption

intervention outcome

re-sprains

\begin{abstract}
A B S T R A C T
Objectives: To study the impact of initial healthcare for a lateral ankle sprain on the outcome of neuromuscular training (NMT) on recurrent sprains and describe athlete characteristics receiving different types of healthcare.

Design: Secondary analysis of three randomized trials.

Method: From three previous trials evaluating NMT, data on athlete characteristics, type of initial healthcare and a recurrent sprain during one-year follow-up were collected $(\mathrm{N}=705)$. Multilevel logistic regression analyses were used to test the impact of initial healthcare on the outcome of NMT on recurrent sprains. Potential differences in athlete characteristics between different types of healthcare were examined using one-way analysis of variance and Pearson chi-square test.

Results: After NMT, 39.7\% of the athletes visiting paramedical care reported a recurrent sprain, $21.8 \%$ of secondary and $34.0 \%$ of primary care. Athletes who visited a physiotherapist reported more recurrent sprains, than those not visiting a physiotherapist (adjusted OR 3.15;95\%CI 1.88-5.23). Athletes visiting paramedical (49.7\%) and primary care (48.4\%) used more braces and/or tape during sport than those not visiting any care (34.2\%).

Conclusions: The initial type of healthcare of athletes with an ankle sprain, that consecutively received NMT, seems to impact the occurrence of recurrent sprains. Physiotherapists may be visited by athletes with a poorer prognosis, which may be explained by different athlete characteristics.
\end{abstract}

(c) 2020 Sports Medicine Australia. Published by Elsevier Ltd. All rights reserved.
Practical implications

- The initial type of healthcare for a lateral ankle sprain among athletes, that were thereafter treated with NMT, seems to impact the occurence of recurrent sprains.

- Physiotherapists should be aware that they are likely to see a specific population of ankle sprain patients thay may have a poorer prognosis, i.e. have a higher risk on sustaining a recurrent sprain.

\section{Introduction}

Ankle sprains are one of the most common traumas to the musculoskeletal system. In the United States the incidence rate is 2.15 per 1000 person-years in the general population and 3.29 per 1000

\footnotetext{
* Corresponding author.

E-mail address: a.mailuhu@erasmusmc.nl (A.K.E. Mailuhu).
}

person-years in patients attending an emergency department. ${ }^{1,2}$ After sustaining an ankle sprain, up to $33 \%$ of the patients still experience persisting complaints like pain, swelling and stiffness after 12 months. ${ }^{3}$ Even after five-years almost $20 \%$ of the patients report persistent complaints. ${ }^{4}$ In addition to persisting complaints, an increased risk of a recurrent sprain is especially present in the first year after the index sprain. ${ }^{3}$ Studies with long-term follow-up (5-7 years) showed that after an index sprain up to $20 \%$ reported recurrent injuries. ${ }^{5,6}$

Thus, an ankle sprain has long-lasting impact on physical function and the use of medical care, up to 12 months after the injury. ${ }^{7}$ Therefore, these injuries do not only pose a physical burden for the individual, but also bring a societal financial burden. Even in a small country like the Netherlands (about 17 million inhabitants), the annual medical costs for ankle sprains among athletes who attend the emergency room was in 2017 almost $€ 6$ million. ${ }^{8}$

Provided the individual and societal burden after an ankle sprain, there is demand for effective and evidence-based treatment 
Table 1

Characteristics of trials included.

\begin{tabular}{|c|c|c|c|}
\hline & Hupperets et al. (2009)* & Janssen et al. $(2014)^{\dagger}$ & Reijen et al. (2017) \\
\hline Total trial sample size $(\mathrm{n})$ & 522 & 384 & 220 \\
\hline Received neuromuscular training program (n) & 258 & 227 (with $n=120$ in combination with a brace) & 220 \\
\hline Age (years), mean (SD) & $28.6(11.8)$ & $34.1(13.4)^{5}$ & $37.8(13.4)$ \\
\hline Sex (male), n (\%) & $136(52.7)$ & $110(48.5)$ & $110(50.0)$ \\
\hline BMI $\left(\mathrm{kg} / \mathrm{m}^{2}\right)$, mean (SD) & $23.4(3.2)^{\|}$ & $23.8(3.2)^{9}$ & $23.2(2.8)$ \\
\hline
\end{tabular}

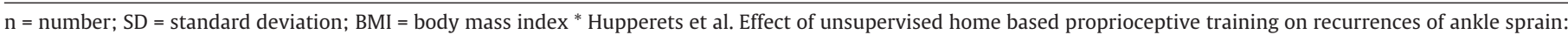

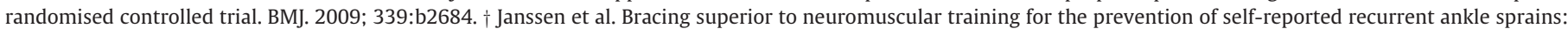

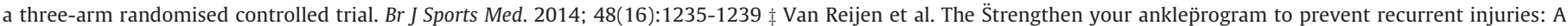
randomized controlled trial aimed at long-term effectiveness. J Sci Med Sport. 2017; 20(6):549-554 §, II, n = 1 unknown

and prevention modalities. One of such is neuromuscular training (NMT), which has been proven effective in preventing recurrent sprains after an initial ankle sprain.9,10 Our group performed three randomized trials in the Netherlands, all including a NMT intervention arm. ${ }^{11-13}$ Hupperets et al. ${ }^{11}$ were the first to show that the NMT was effective but also showed that NMT was specifically beneficial in athletes whose index ankle sprain was self-treated rather than by a healthcare provider. This raises the questions whether there is an association between the healthcare provider who treated the index sprain initially and the effect of NMT on recurrent ankle sprains and,also, whether athlete characteristics differ between the different type of healthcare providers. These characteristics will be useful for healthcare providers to take into account when choosing intervention strategies or determine prognosis.

Therefore, the aims of this study were to (1) study the impact of the initial type of healthcare for a lateral ankle sprain on the outcome after NMT for the prevention of recurrent sprains and to (2) describe characteristics of athletes visiting different types of healthcare and examine potential differences in these characteristics.

\section{Methods}

The current study presents a post-hoc secondary analysis of the combined individual patient data as collected in three previous Dutch trials on the preventive effects of NMT on recurrent ankle sprains. These trials evaluated, in chronological order (1) the effectiveness of NMT for the prevention of recurrent ankle sprains $(n=522),(2)$ the effectiveness of NMT against bracing without and in combination with NMT $(n=384)$ and (3) the effectiveness of delivering NMT through an interactive mobile application against written exercises materials $(n=220)$ (Table 1$).{ }^{11-13}$ For each study ethical approval was obtained by the medical ethics committee of the VU University Medical Center, Amsterdam, Netherlands. All athletes provided written informed consent prior to inclusion. For athletes under the age of 18 additional parental informed consent was provided.

All three trials included recreational athletes (12-70 years), who had sustained a lateral ankle sprain within two months prior to inclusion (i.e. index ankle sprain). Treatment of the index ankle sprain followed usual care with the healthcare that the athletes had visited on their own initiative. The same NMT was provided in each of the three trials ${ }^{11-13}$ once the athlete entered the study. All studies had a prospective design with a 12-month follow-up. A recurrent sprain was reported by the athlete through monthly questionnaires and defined as a self-reported sudden inversion of the index ankle. For the purpose of the current study we only included those athletes who were allocated to the study groups that received the NMT (Table 1).

Baseline data from the three trials were used to describe athlete characteristics, i.e. demographics (age, gender, body mass index $(B M I))$, occurrence of any ankle sprains before the index sprain ('yes' or 'no'), initial type of healthcare setting that athlete visited for index sprain ('none'; 'paramedical care setting: physiotherapist, manual therapist, exercise therapist'; 'secondary care setting: general surgeon, orthopaedic surgeon'; 'primary care setting: general practitioner (GP), sports physician'; 'other'), initial type of healthcare provider that athlete visited for index sprain (GP, sports physician, physiotherapist), type of sports, occurrence of index ankle sprain during a sport activity ('yes' or 'no') and the regular use of a brace and/or tape during sport activity ('yes, for one ankle'; 'yes, for both ankles' or 'no'). Data from the one year follow-up of each trial were used to register the occurrence of recurrent sprains ('yes' or 'no'), time to recurrent sprain (months) and sports exposure until recurrent sprain (hours).

In order to pool the data of the three individual trials, the variables that were not labeled identical between the trials, were redefined in new variables (Table 2). Only athletes with available data on baseline and follow-up were used for the pooled analyses. The number of athletes from the received original dataset $(n=258)$ of Hupperets et $\mathrm{al}^{11}$ did not correspond with the number of athletes described in the published article $(n=256)$. The reason for the discrepancy could not be retrieved and therefore, the number of athletes in the original dataset was used for the purpose of our study $(\mathrm{n}=258)$.

The primary outcome of interest was the incidence of recurrent sprains during follow-up, reported as the number of self-reported recurrent sprains and as the recurrence incidence density per 1000 hours of sport participation until the occurrence of the recurrent sprain. A recurrent sprain was defined as a self-reported sudden inversion of the index ankle, not necessarily leading to cessation from participation or time-loss.

Descriptive statistics were used to describe athlete characteristics, the types of initial healthcare settings and healthcare providers for the index ankle sprain, the incidence of recurrent sprains and time in months to onset of the reported recurrent sprain, using the mean and standard deviation (SD) for continuous data and proportions for categorical data.

Potential differences in characteristics between athletes visiting different types of initial healthcare settings and between athletes visiting different types of initial healthcare providers for their index ankle sprain were examined using one-way analysis of variance (ANOVA) for continuous variables and Pearson chi-square test for categorical variables. When a significant difference was found, post-hoc Bonferroni correction was performed in order to adjust for multiple comparison.

Multilevel logistic regression analyses were performed to examine the impact of the initial types of healthcare settings and initial types of healthcare provider for a lateral ankle sprain on the outcome of NMT for the prevention of recurrent sprains. Data on visits to specified healthcare provider for the index ankle sprain was only available in 2 trials $(n=447) .{ }^{12,13}$ The different studies were used as cluster level in the multilevel analyses, ${ }^{11-13}$ the initial types of healthcare setting and providers were used as independent variables, and the occurrence of a recurrent sprain during follow-up was used as dependent variable. Analyses were adjusted 
Table 2

Definitions of redefined variables for pooling data of trials included.

\begin{tabular}{|c|c|c|c|c|}
\hline Redefined variables & Definition by Hupperets et al.* & Definition by Janssen et al. ${ }^{\dagger}$ & Definition by Reijen et al. ${ }^{\ddagger}$ & $\begin{array}{l}\text { Definition of variable in pooled } \\
\text { data }\end{array}$ \\
\hline \multicolumn{5}{|l|}{ Baseline variables } \\
\hline $\begin{array}{l}\text { Previous sprain before index } \\
\text { ankle sprain }\end{array}$ & $\begin{array}{l}\text { Previous sprain before } \\
\text { inclusion ankle sprain } \\
\text { 'yes' or 'no' }\end{array}$ & $\begin{array}{l}\text { Previous sprain before } \\
\text { inclusion ankle sprain } \\
\text { 'yes' or 'no' }\end{array}$ & $\begin{array}{l}\text { Previous sprain before } \\
\text { inclusion ankle sprain } \\
\text { 'no'; 'yes incidental } 2 \text { or more' } \\
\text { or 'yes frequent, } 3 \text { or more' }\end{array}$ & $\begin{array}{l}\text { 'no' } \\
\text { 'yes' (including 'yes incidental } \\
\text { ( } 2 \text { or less)' and 'yes frequent ( } 3 \\
\text { or more)') }\end{array}$ \\
\hline $\begin{array}{l}\text { Regular use of brace and/or } \\
\text { tape during sport activity }\end{array}$ & $\begin{array}{l}\text { Use of brace during sport } \\
\text { activity 'yes on both ankles'; 'yes } \\
\text { on left ankle'; 'yes on right ankle' } \\
\text { or 'no' } \\
\text { Use of tape during sport } \\
\text { activity 'yes on both ankles'; 'yes } \\
\text { on left ankle'; 'yes on right ankle' } \\
\text { or 'no' }\end{array}$ & $\begin{array}{l}\text { Use of brace or tape 'none', } \\
\text { 'brace', 'tape' or 'both' }\end{array}$ & $\begin{array}{l}\text { Use of brace during sport } \\
\text { activity 'yes on both ankles'; 'yes } \\
\text { on injured ankle'; 'yes on } \\
\text { non-injured ankle' or 'no' } \\
\text { Use of tape during sport } \\
\text { activity 'yes on both ankles'; 'yes } \\
\text { on injured ankle'; 'yes on } \\
\text { non-injured ankle' or 'no' }\end{array}$ & $\begin{array}{l}\text { Data on brace and tape pooled: } \\
\text { 'no' 'yes, on both ankles' or 'yes, } \\
\text { on one ankle' (including 'yes on } \\
\text { left ankle'; 'yes on right ankle'; } \\
\text { 'yes on injured ankle' and 'yes on } \\
\text { non-injured ankle') }\end{array}$ \\
\hline $\begin{array}{l}\text { Initial type of healthcare } \\
\text { setting for index ankle sprain }\end{array}$ & $\begin{array}{l}\text { Treatment for inclusion ankle } \\
\text { sprain 'no medical treatment'; } \\
\text { 'paramedical treatment: } \\
\text { physiotherapist, manual } \\
\text { therapist, exercise therapist'; } \\
\text { 'intramural treatment: general } \\
\text { surgeon, orthopeadic surgeon, } \\
\text { emergency physician' or } \\
\text { 'extramural treatment: general } \\
\text { practitioner, sports physician' }\end{array}$ & $\begin{array}{l}\text { Treatment for inclusion ankle } \\
\text { sprain 'none', 'sport masseur', } \\
\text { 'trainer', 'general practitioner', } \\
\text { 'physiotherapist', 'sports } \\
\text { physician', 'orthopeadic } \\
\text { surgeon', 'general surgeon', } \\
\text { 'emergency physician' or 'other' }\end{array}$ & $\begin{array}{l}\text { Treatment for inclusion ankle } \\
\text { sprain 'no treatment', 'sports } \\
\text { physician', 'physiotherapist', } \\
\text { 'sport masseur', general } \\
\text { practitioner', 'sports } \\
\text { physiotherapist' or 'other' }\end{array}$ & $\begin{array}{l}\text { 'none' 'paramedical care setting: } \\
\text { physiotherapist, manual } \\
\text { therapist, exercise therapist' } \\
\text { 'secondar care setting: general } \\
\text { surgeon, orthopeadic surgeon, } \\
\text { emergency physician' 'primary } \\
\text { care setting: general } \\
\text { practitioner, sports physician' or } \\
\text { 'other' }\end{array}$ \\
\hline $\begin{array}{l}\text { Initial type of healthcare } \\
\text { provider for index ankle } \\
\text { sprain }\end{array}$ & Not available & $\begin{array}{l}\text { Treatment for inclusion ankle } \\
\text { sprain 'none', 'sport masseur', } \\
\text { 'trainer', 'general practitioner', } \\
\text { 'physiotherapist', 'sports } \\
\text { physician', 'orthopeadic } \\
\text { surgeon', 'general surgeon', } \\
\text { 'emergency physician' or 'other' }\end{array}$ & $\begin{array}{l}\text { Treatment for inclusion ankle } \\
\text { sprain 'no treatment', 'sports } \\
\text { physician', 'physiotherapist', } \\
\text { 'sport masseur', general } \\
\text { practitioner', 'sports } \\
\text { physiotherapist' or 'other' }\end{array}$ & $\begin{array}{l}\text { 'general practitioner', 'sports } \\
\text { physician' or 'physiotherapist' }\end{array}$ \\
\hline
\end{tabular}

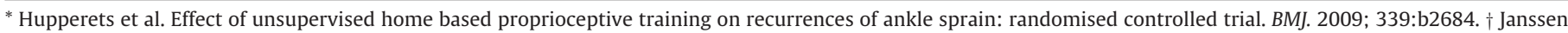

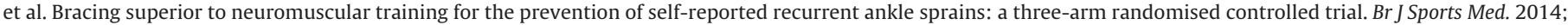

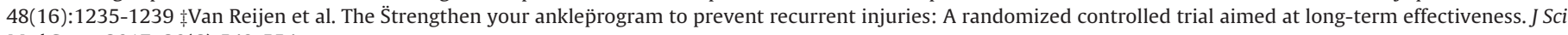
Med Sport. 2017; 20(6):549-554

for potential confounders (i.e. athlete characteristics) that were determined from the literature or based on a priori assumptions, including the use of brace in addition to NMT in athletes from trial of Janssen et $\mathrm{al}^{12}(\mathrm{n}=120)$ and sports exposure (hours) until recurrent sprain. ${ }^{14,15}$ Results of the regression analyses were reported in unadjusted and adjusted odds ratios (OR) and their 95\% confidence interval $(\mathrm{CI})$.

The significance level was set on 0.05 and all analyses were performed with the Statistical Package for the Social Sciences V. 21.0. (SPSS, IBM, Armonk, NY).

\section{Results}

A total of 705 athletes who received NMT, were included in this study. Athletes from the trial of van Reijen et al. ${ }^{13}$ were significantly older (37.8 (SD 13.4) years) than the athletes of the trial of Janssen et al. ${ }^{12}$ (34.1 (SD 13.4) years) and of the trial of Hupperets et al. ${ }^{11}$ (28.6 (SD 11.8) years) (Table 1 ). The mean age of our population was 33.3 (SD 13.4) years (Table 3). A previous ankle sprain was reported in $70.1 \%$ of the athletes and $76 \%$ of the index ankle sprains occurred during a sport activity.

The distribution of the initial type of healthcare setting that the athletes visited for their index sprain is presented in Fig. 1. The type of healthcare setting was unknown in $8.5 \%$ of the study population. Since only one athlete reported an 'other' healthcare setting, data of this subject were excluded for further analyses. A large part of the athletes $(n=238 ; 37.0 \%)$ did not visit a healthcare provider for their index ankle sprain, and of those athletes who did, most $(n=257$; $39.9 \%$ ) visited a paramedical care setting.

A total of 252 (35.7\%) recurrent sprains were reported during one-year follow-up, with an overall recurrence incidence density
Table 3

Baseline athlete characteristics

\begin{tabular}{ll}
\hline & $\begin{array}{l}\text { Total study } \\
\text { population } \\
\mathrm{n}=705\end{array}$ \\
\hline Athlete characteristics & \\
Age (years), mean (SD) & $33.3(13.4)^{*}$ \\
Sex (male) & $356(50.5)$ \\
BMI (kg/m²), mean (SD) & $23.4(3.1)^{\dagger}$ \\
Previous ankle sprain before index ankle sprain & $493(70.1)^{\ddagger}$ \\
Top 3 sport activities & \\
1. Athletics/running & $137(19.4)$ \\
2. Soccer & $117(16.6)$ \\
3. Volleyball & $94(13.4)$ \\
Regular use of brace and/or tape during sport activity $\|$ & $231(44.4)^{\ddagger}$ \\
Occurrence of index ankle sprain during sport activity & $534(76.0)^{\#}$ \\
\hline
\end{tabular}

Presented in $\mathrm{n}(\%)$, unless otherwise stated $\mathrm{n}=$ number; $\mathrm{SD}=$ standard deviation; $\mathrm{BMI}$ = body mass index $* \mathrm{n}=1$ unknown $; \nmid \mathrm{n}=2$ unknown $; \ddagger \mathrm{n}=2$ unknown $; \boldsymbol{\uparrow} \mathrm{n}=185$ unknown; \# $n=60$ unknown $\S$ In population of $n=704$, since one athlete reported not participating in sport at time of inclusion || Including data on the regular use of brace and/or tape during sport activity on one ankle and both ankles

of 3.98 per 1000 hours of sports participation (95\%CI 3.49-4.47). Almost $40 \%$ of the athletes who visited paramedical care setting reported a recurrent sprain (39.7\%), with an injury incidence density of 4.11 per 1000 hours of sports participation (95\%CI 3.31-4.91) (Fig. 2). Athletes visiting a secondary care setting had a mean time to a recurrent sprain of 7.1 months (SD 3.0), whereas athletes visiting other care settings and other healthcare providers had a mean time to a recurrent sprain variating from 2.0 to 4.0 months.

Multilevel logistic regression analyses on the impact of the initial type of healthcare provider on the outcome of NMT on ankle sprain recurrences, showed more recurrent sprains after NMT in 
Total population $n=705$

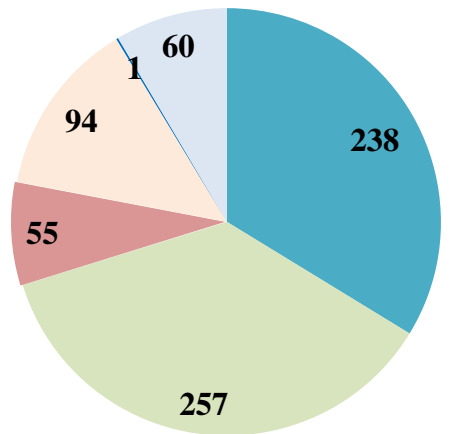

\section{No healthcare \\ Paramedical care \\ - Secondary care \\ Primary care}

- Other treatment

Unkown

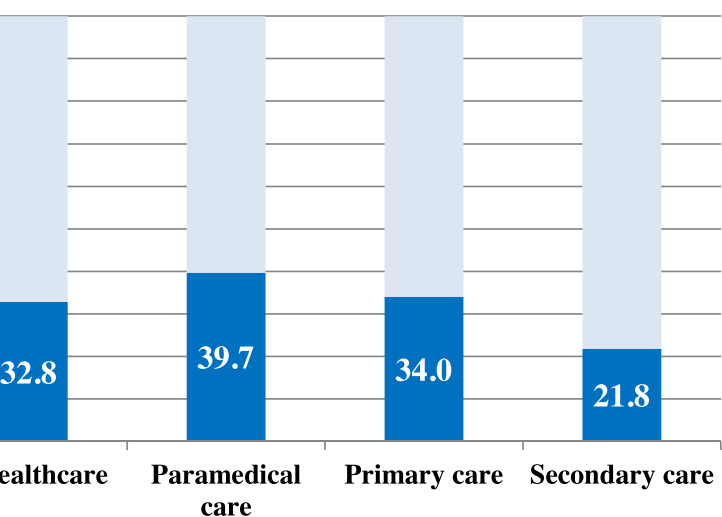

\section{No recurrent}

sprain

\section{Recurrent}

sprain

Fig. 2. Number of recurrent sprains during one-year follow-up (\%).

Table 4

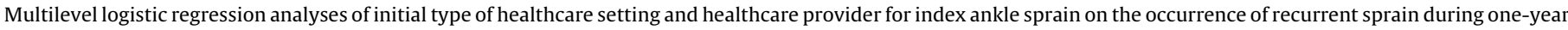
follow-up

\begin{tabular}{|c|c|c|c|c|c|c|c|}
\hline \multicolumn{4}{|l|}{ Total study population $\mathrm{N}=705^{*}$} & \multicolumn{4}{|c|}{$\begin{array}{l}\text { Multilevel logistic regression analyses of type of } \\
\text { healthcare setting on occurrence of recurrent sprain }\end{array}$} \\
\hline $\begin{array}{l}\text { Initial type of healthcare } \\
\text { setting }\end{array}$ & $\begin{array}{l}\text { Recurrent } \\
\text { sprain during } \\
\text { follow-up }\end{array}$ & $\begin{array}{l}\text { Incidence of recurrent } \\
\text { sprains }(95 \% \mathrm{CI}) \text { per } \\
1000 \text { hours of sport } \\
\text { participation }\end{array}$ & $\begin{array}{l}\text { Mean time to } \\
\text { recurrent sprain in } \\
\text { months }(S D)\end{array}$ & $\begin{array}{l}\text { Unadjusted OR } \\
(95 \% \mathrm{CI})\end{array}$ & p-value & $\begin{array}{l}\text { Adjusted } \mathrm{OR}^{\ddagger}(95 \% \\
\mathrm{CI})\end{array}$ & p-value \\
\hline No healthcare setting $(n=238)$ & $78(32.8)$ & $3.51(2.73-4.29)$ & $4.0(3.4)$ & $1.03(0.84-1.26)$ & 0.77 & $0.96(0.77-1.20)$ & $0.74^{\S}$ \\
\hline $\begin{array}{l}\text { Paramedical care setting } \\
\text { (physiotherapist, manual } \\
\text { therapist, exercise therapist) } \\
(n=257)\end{array}$ & $102(39.7)$ & $4.11(3.31-4.91)$ & $3.9(3.5)$ & $1.09(0.95-1.26)$ & 0.22 & $1.28(0.94-1.74)$ & $0.12^{\|}$ \\
\hline $\begin{array}{l}\text { Secondary care setting (general } \\
\text { surgeon, orthopaedic surgeon } \\
\text { emergency physician) }(n=55)\end{array}$ & $12(21.8)$ & $1.99(0.87-3.12)$ & $7.1(3.0)$ & $0.68(0.28-1.66)$ & 0.40 & $0.87(0.47-1.60)$ & $0.65^{\pi}$ \\
\hline $\begin{array}{l}\text { Primary care setting (general } \\
\text { practitioner, sports physician) } \\
(n=94)\end{array}$ & $32(34.0)$ & $4.79(3.13-6.45)$ & $3.0(2.9)$ & $0.86(0.48-1.55)$ & 0.62 & $0.82(0.37-1.84)$ & $0.63^{\#}$ \\
\hline Unknown $(n=60)$ & $27(45.0)$ & $7.78(4.85-10.72)$ & $3.2(3.0)$ & - & - & - & - \\
\hline \multicolumn{4}{|l|}{ Total study population $\mathrm{N}=447^{* *}$} & \multicolumn{4}{|c|}{$\begin{array}{l}\text { Multilevel logistic regression analyses of type of } \\
\text { healthcare provider on occurrence of recurrent sprain }\end{array}$} \\
\hline $\begin{array}{l}\text { Initial type of healthcare } \\
\text { provider }\end{array}$ & $\begin{array}{l}\text { Recurrent } \\
\text { sprain during } \\
\text { follow-up }\end{array}$ & $\begin{array}{l}\text { Incidence of recurrent } \\
\text { sprains }(95 \% \mathrm{CI}) \text { per } \\
1000 \text { hours of sport } \\
\text { participation }\end{array}$ & $\begin{array}{l}\text { Mean time to } \\
\text { recurrent sprain in } \\
\text { months }(\mathrm{SD})\end{array}$ & $\begin{array}{l}\text { Unadjusted OR } \\
(95 \% \mathrm{CI})\end{array}$ & p-value & $\begin{array}{l}\text { Adjusted } \mathrm{OR}^{\ddagger}(95 \% \\
\mathrm{CI})\end{array}$ & p-value \\
\hline General practitioner $(n=70)$ & $23(32.9)$ & $5.01(2.96-7.05)$ & $2.0(1.4)$ & $0.84(0.40-1.79)$ & 0.65 & $0.77(0.08-7.15)$ & 0.81 \\
\hline Physiotherapist $(\mathrm{n}=118)$ & $50(42.4)$ & $4.43(3.20-5.66)$ & $3.2(3.0)$ & $1.58(1.09-2.28)$ & 0.02 & $3.15(1.88-5.23)$ & $<0.01$ \\
\hline Sports physician $(n=11)^{\dagger \dagger}$ & $4(36.4)$ & $4.77(0.10-9.45)$ & $2.0(0.8)$ & - & - & - & - \\
\hline
\end{tabular}

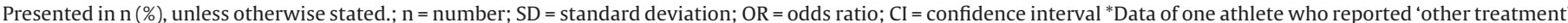

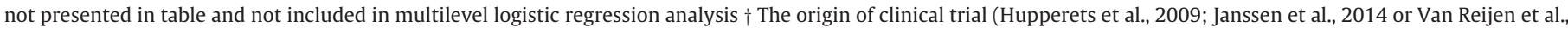

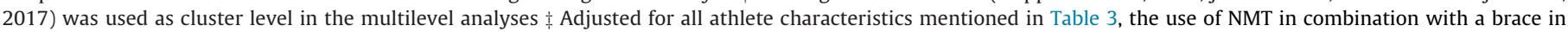

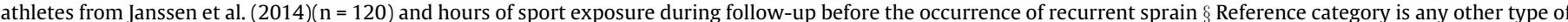

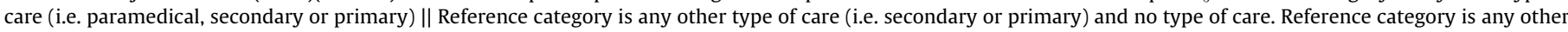

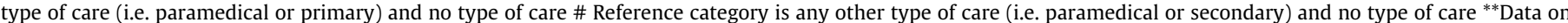

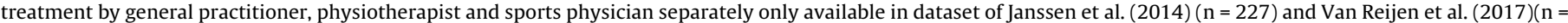
220) $\dagger \dagger$ Group excluded in multilevel logistic regression analyses due to small number of $n=11$ 
Table 5

Athlete characteristics by initial type of healthcare setting and healthcare provider for index ankle sprain

\begin{tabular}{|c|c|c|c|c|c|c|c|}
\hline \multicolumn{8}{|l|}{ Total study population $n=705^{*}$} \\
\hline $\begin{array}{l}\text { Initial type of healthcare } \\
\text { setting }\end{array}$ & $\begin{array}{l}\text { Number of athletes } \\
\text { visiting type of } \\
\text { healthcare setting }\end{array}$ & $\begin{array}{l}\text { Age (years), } \\
\text { mean }(S D)\end{array}$ & Sex (male) & $\begin{array}{l}\text { BMI }\left(\mathrm{kg} / \mathrm{m}^{2}\right) \text {, } \\
\text { mean }(\mathrm{SD})\end{array}$ & $\begin{array}{l}\text { Previous sprain } \\
\text { before index } \\
\text { ankle sprain }\end{array}$ & $\begin{array}{l}\text { Regular use of } \\
\text { brace and/or tape } \\
\text { during sport } \\
\text { activity }\end{array}$ & $\begin{array}{l}\text { Occurrence of } \\
\text { index ankle } \\
\text { sprain during } \\
\text { sport activity }\end{array}$ \\
\hline No healthcare setting & $238(37.0)$ & $32.3(13.1)$ & $130(54.6)$ & $23.6(3.1)$ & $168(70.6)$ & $64(34.2)^{\dagger}$ & $166(69.7)$ \\
\hline $\begin{array}{l}\text { Paramedical care setting } \\
\text { (physiotherapist, manual } \\
\text { therapist, exercise therapist) }\end{array}$ & $257(39.9)$ & $32.1(13.3)^{\ddagger}$ & $128(49.8)$ & $23.0(2.9)^{\S}$ & $179(69.6)$ & $88(49.7)^{\|}$ & $205(79.8)$ \\
\hline $\begin{array}{l}\text { Secondary care setting (general } \\
\text { surgeon, orthopaedic surgeon } \\
\text { emergency physician) }\end{array}$ & $55(8.5)$ & $31.5(13.4)$ & $23(41.8)$ & $23.8(4.2)$ & $37(67.3)$ & $30(55.6)^{\natural}$ & $44(80.0)$ \\
\hline $\begin{array}{l}\text { Primary care setting (general } \\
\text { practitioner, sports physician) }\end{array}$ & $94(14.6)$ & $35.5(12.5)$ & $44(46.8)$ & $23.6(2.8)$ & $62(66.0)$ & $31(48.4)^{\#}$ & $80(85.1)$ \\
\hline Unknown** & $60(8.5)$ & $40.4(14.2)$ & $31(51.7)$ & $23.9(2.8)$ & $46(79.3)^{\dagger \dagger}$ & $18(48.6)^{\ddagger \ddagger}$ & $38(65.5)^{53}$ \\
\hline p-value ${ }^{|\||}$ & - & 0.13 & 0.28 & 0.12 & 0.85 & $<0.01^{\text {ศ }}$ & $<0.01^{\# \#}$ \\
\hline \multicolumn{8}{|l|}{ Total study population $n=447^{* * *}$} \\
\hline $\begin{array}{l}\text { Initial type of healthcare } \\
\text { provider }\end{array}$ & $\begin{array}{l}\text { Number of athletes } \\
\text { visiting type of } \\
\text { healthcare } \\
\text { provider }\end{array}$ & $\begin{array}{l}\text { Age (years), } \\
\text { mean }(S D)\end{array}$ & Sex (male) & $\begin{array}{l}\text { BMI }\left(\mathrm{kg} / \mathrm{m}^{2}\right) \text {, } \\
\text { mean }(\mathrm{SD})\end{array}$ & $\begin{array}{l}\text { Previous sprain } \\
\text { before index } \\
\text { ankle sprain }\end{array}$ & $\begin{array}{l}\text { Regular use of } \\
\text { brace and/or tape } \\
\text { during sport } \\
\text { activity }\end{array}$ & $\begin{array}{l}\text { Occurrence of } \\
\text { index ankle } \\
\text { sprain during } \\
\text { sport activity }\end{array}$ \\
\hline General practitioner & $70(15.7)$ & $35.6(12.1)$ & $29(41.4)$ & $23.7(2.7)$ & $48(68.8)$ & $26(52.0)^{\dagger \dagger \dagger}$ & $62(88.6)$ \\
\hline Physiotherapist & $118(26.4)$ & $33.9(13.3)^{\ddagger \ddagger}$ & $59(50.0)$ & $23.4(2.6)^{\S \S \S}$ & $87(73.7)$ & $47(58.8)$ ตศศ & $95(80.5)$ \\
\hline Sports physician & $11(2.5)$ & $38.5(12.3)$ & $7(63.6)$ & $22.6(1.9)$ & $6(54.5)$ & $2(100)^{\# \# \#}$ & $10(90.9)$ \\
\hline p-value $e^{* * * *}$ & - & 0.42 & 0.29 & 0.34 & 0.36 & 0.35 & 0.28 \\
\hline
\end{tabular}

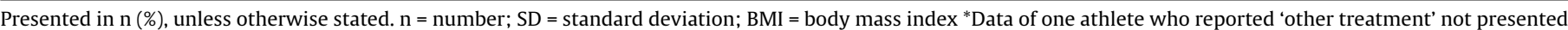

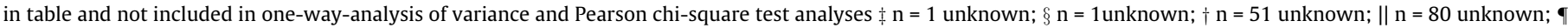

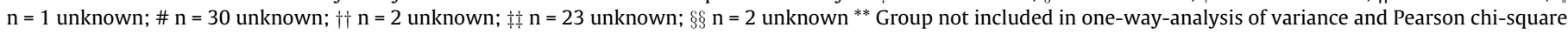

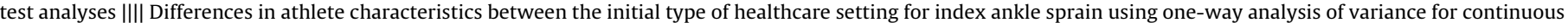
variables and Pearson chi-square test for categorical variables.

'ศ Post hoc significant difference between 'none' and 'paramedical care setting' and between 'none' and 'secondary care setting' ( $\mathrm{p}<0.05$ ).

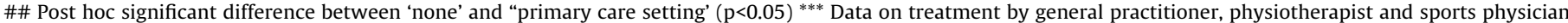

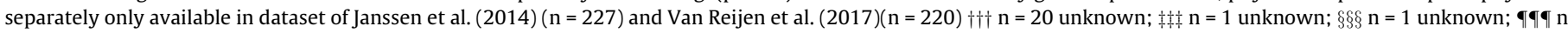

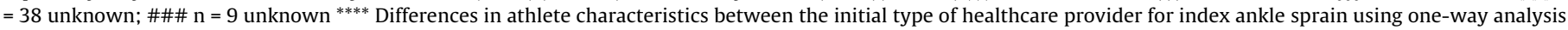
of variance for continuous variables and Pearson chi-square test for categorical variables.

athletes that visited a physiotherapist for their initial index ankle sprain when compared to those who had not visited a physiotherapist (adjusted OR 3.15; 95\%CI 1.88-5.23) (Table 4). No significant differences on the occurrence of recurrent sprains were found between the different types of healthcare settings on the outcome of NMT.

Characteristics of athletes visiting different types of healthcare settings and different types of healthcare providers for their initial index ankle sprain are presented in Table 5. There were no significant differences in characteristics between the group of athletes, with missing data on the initial type of healthcare setting and the group of athletes with available data. Significantly more athletes who visited paramedical or secondary care setting used a brace and/or tape regularly during sport activity (49.7\% and 55.6\% respectively), than those who did not visit any healthcare provider (34.2\%) $(\mathrm{p}<0.01)$. More often in athletes who visited primary care setting, the index ankle sprain had occurred during sports activities (85.1\%) when compared to those who did not visit any care setting $(69.7 \%)(p<0.01)$. No further differences were found in other athlete characteristics, nor between the different healthcare providers GP, physiotherapist and sports physician.

\section{Discussion}

We pooled data of 705 athletes from three trials to examine the impact of initial type of healthcare for a lateral ankle sprain on ankle sprain recurrences during one-year follow-up after NMT. 252 athletes $(37.5 \%)$ reported a recurrent sprain during follow-up, which is comparable with previous studies evaluating recurrent sprains during follow-up. ${ }^{3,16}$ More recurrent sprains occurred in athletes who visited a physiotherapist compared to those who did not visit a physiotherapist. The use of braces and/or tape and the occurrence of the index sprain during sports activities were characteristics that significantly differed between initial type of healthcare settings.

Although we found a significant difference in the occurrence of recurrent sprains between athletes visiting different healthcare providers in a subsample of the study, we found no differences in the occurrence of recurrent sprains when we clustered health care settings. However, we did see a positive trend towards more recurrent sprains in athletes visiting paramedical care setting, than those visiting other types of care setting. This may be explained by the inclusion of physiotherapists in the paramedical care setting category. The fact that we found a significant difference between athletes visiting a physiotherapist and those who did not visit a physiotherapist, is likely the result of selection bias. Athletes that visited a physiotherapist for their index ankle sprain are likely to differ from those visiting other healthcare providers in their characteristics or preference for the type of healthcare provider. Some of the unmeasured variables, including injury severity and activity level, may differ between groups. Moreover, the data showed that athletes visiting the physiotherapist seem to be more likely to have had a previous sprain before their index ankle sprain. Therefore, athletes visiting the physiotherapist may already be at a higher risk of sustaining a recurrent sprain before receiving treatment. No information was available on the reason for choosing a particular healthcare provider in our datasets. It can therefore only be hypothesized that the type of healthcare provider, i.e. the physiotherapist, is likely to serve as a proxy variable in our dataset: it might not itself directly explain our result, but might serves in place of a variable, i.e. athlete characteristic, that was not available in our study. 
No differences were found in characteristics between the athletes visiting different healthcare providers, but we did find differences in characteristics of athletes visiting different types of healthcare settings. Athletes visiting paramedical care setting and visiting secondary care setting used significantly more braces and/or tape regularly during sport activities than athletes who did not seek any care setting. Potentially, the use of a brace or tape was applied by the paramedical or secondary physician, as part of the rehabiliation of the index ankle sprain. However, it may also be hypothesized that these athletes, who chose to visit a paramedical or secondary care setting, perhaps sustained a more severe injury or participated in a high-risk sport, for which a brace or tape was preferred. We had no information on why and when the athletes started to use a brace and/or tape. In the literature, little is known about characteristics of patients attending different healthcare settings and providers. Only one recent study described characteristics of patients attending the emergency department in the UK.17 This study found that more younger men (14-37 years old) than women of the same age visited the ER with an ankle injury. However, they made no comparison with other healthcare settings. Therefore, our findings are difficult to compare with existing literature.

To our knowledge, this is the first study evaluating the impact of initial type of healthcare for a lateral ankle sprain on the outcome of NMT on recurrent sprainrisk and describing characteristics of athletes attending different types of healthcare. A strength is that all included athletes received the same NMT and had one-year of follow-up. We think this period is appropriate to detect rcurrent sprains after the index ankle sprain, as the risk for a recurrent sprain is increased during the first year after an initial sprain. ${ }^{18,19}$ Also, we believe that we had information on a representative set of types of healthcare settings and providers, in both primary and secondary care, that are involved in the treatment of an ankle sprain..$^{9,10}$

Nevertheless, some limitations should be addressed. For the purpose of our study we focused on the initial type of healthcare setting and provider and we did not focus on the specific type of treatment that was given by the involved healthcare provider. Different types of healthcare providers can apply similar treatment modalities (e.g. both physiotherapist and sports physician can apply a type of exercise therapy) and also, one type of healthcare provider can apply different types of treatment modalities (e.g. a GP can apply functional or exercise therapy). It would be interesting to evaluate if the outcome of the type of treatment differs between different types of healthcare provider. Another limitation, is the fact that data on the separate healthcare providers were only available in two trials. ${ }^{12,13}$ As a consequence, we presented a smaller proportion of athletes visiting the different healthcare providers (GP, physiotherapist, sports physician). Thus, we might have found a smaller impact of the healthcare provider for a lateral ankle sprain on the outcome after NMT on recurrent sprains than the true impact and we might not detected existing differences between characteristics of athletes visiting the separate healthcare providers. Moreover, for $8.5 \%$ of the subjects the type of healthcare setting was unknown and these subjects were therefore not included in the multilevel regression analyses. Lastly, only athletes were included in our study and it is known that sports participants are at a higher risk for recurrent ankle sprains than non-athletes. ${ }^{9,16}$ Therefore, our results are not generalizable to a general population which includes non-athletes. However, since ankle sprains are a very common injury among sporting populations, ${ }^{17,20}$ a first evaluation of the impact of initial healthcare for a lateral ankle sprain in this population was a useful approach.

From our study it seems that specifice athlete characteristics are associated with a visit to specific healthcare providers. Consequently, this seems to influence the outcome of NMT as evaluated in our study. This should add towards awareness of physiotherapists that they are likely to see a specific population that may have a poorer prognosis, i.e. have higher risk on sustaining a recurrent sprain. We think that our study gives insight in the pathway of healthcare consumption of ahtletes with an ankle sprain and its impact on recurrent sprains after NMT. Further research with larger sample sizes is necesarry to evaluate the impact of initial healthcare on the prognosis of ankle sprains.

\section{Conclusion}

Among athletes that were treated with NMT, the initial type of healthcare for an ankle sprain, seems to impact the occurrence of recurrent sprains. According to our study, physiotherapists may be visited by athletes with a poorer prognosis and should take this into account when determining rehabilitation strategies. This seems to be due to different characteristics of athletes visiting different types of healthcare.

\section{Acknowledgement}

This study was supported by the Netherlands Organisation for Health Research and Development (ZonMW) [grant number 4201.1007].

\section{Appendix A. Supplementary data}

Supplementary material related to this article can be found, in the online version, at doi:https://doi.org/10.1016/j.jsams.2020.03. 010 .

\section{References}

1. Waterman BR, Owens BD, Davey S, Zacchilli MA, Belmont Jr PJ. The epidemiology of ankle sprains in the United States. J Bone Joint Surg Am 2010; 92(13):2279-2284.

2. Shah S, Thomas AC, Noone JM, Blanchette CM, Wikstrom EA. Incidence and Cost of Ankle Sprains in United States Emergency Departments. Sports Health 2016; 8(6):547-552.

3. van Rijn RM, van Os AG, Bernsen RM, Luijsterburg PA, Koes BW, Bierma-Zeinstra SM. What is the clinical course of acute ankle sprains? A systematic literature review. Am J Med 2008; 121(4), 324-331 e326.

4. Mailuhu AKE, Oei EHG, van Putte-Katier N et al. Clinical and radiological predictors for persistent complaints five years after a lateral ankle sprain: A long-term follow-up study in primary care. J Sci Med Sport 2018; 21(3):250-256.

5. Kemler E, Thijs KM, Badenbroek I, van de Port IG, Hoes AW, Backx FJ. Longterm prognosis of acute lateral ankle ligamentous sprains: high incidence of recurrences and residual symptoms. Fam Pract 2016; 33(6):596-600.

6. Konradsen L, Bech L, Ehrenbjerg M, Nickelsen T. Seven years follow-up after ankle inversion trauma. Scand J Med Sci Sports 2002; 12(3):129-135.

7. van Ochten JM, B-ZS, Bindels PJE, van Middelkoop M. Impact of a Lateral Ankle Sprain in General Practice: Comparison Between Patients With and Without Persistent Complaints After6-12 Months. JSM Foot Ankle 2016; 1(3):1013.

8. Letsel Informatie Systeem. V. Letsellastmodel 2017, Veiligheid NL, 2017.

9. Vuurberg G, Hoorntje A, Wink LM et al. Diagnosis, treatment and prevention of ankle sprains: update of an evidence-based clinical guideline. Br J Sports Med 2018; 52(15):956.

10. Doherty C, Bleakley C, Delahunt E, Holden S. Treatment and prevention of acute and recurrent ankle sprain: an overview of systematic reviews with metaanalysis. Br J Sports Med 2017; 51(2):113-125.

11. Hupperets MD, Verhagen EA, van Mechelen W. Effect of unsupervised home based proprioceptive training on recurrences of ankle sprain: randomised controlled trial. BMJ 2009; 339:b2684.

12. Janssen KW, van Mechelen W, Verhagen EA. Bracing superior to neuromuscular training for the prevention of self-reported recurrent ankle sprains: a three-arm randomised controlled trial. Br J Sports Med 2014; 48(16):1235-1239.

13. Van Reijen M, Vriend I, Zuidema V, van Mechelen W, Verhagen EA. The S̈trengthen your anklep̈rogram to prevent recurrent injuries: A randomized controlled trial aimed at long-term effectiveness. J Sci Med Sport 2017; 20(6):549-554

14. Pourkazemi F, Hiller CE, Raymond J, Black D, Nightingale EJ, Refshauge KM. Predictors of recurrent sprains after an index lateral ankle sprain: a longitudinal study. Physiotherapy 2017.

15. Thompson JY, Byrne C, Williams MA, Keene DJ, Schlussel MM, Lamb SE. Prognostic factors for recovery following acute lateral ankle ligament sprain: a systematic review. BMC Musculoskelet Disord 2017; 18(1):421.

16. Attenborough AS, Hiller CE, Smith RM, Stuelcken M, Greene A, Sinclair PJ. Chronic ankle instability in sporting populations. Sports Med 2014; 44(11):1545-1556. 
17. Al Bimani SA, Gates LS, Warner M, Ewings S, Crouch R, Bowen C. Characteristics of patients with ankle sprain presenting to an emergency department in the south of England (UK): A seven-month review. Int Emerg Nurs 2018.

18. Verhagen EA, Van der Beek AJ, Bouter LM, Bahr RM, Van Mechelen W. A one season prospective cohort study of volleyball injuries. Br J Sports Med 2004; 38(4):477-481.
19. Bahr R, Bahr IA. Incidence of acute volleyball injuries: a prospective cohort study of injury mechanisms and risk factors. Scand J Med Sci Sports 1997; 7(3): 166-171.

20. Doherty C, Delahunt E, Caulfield B, Hertel J, Ryan J, Bleakley C. The incidence and prevalence of ankle sprain injury: a systematic review and meta-analysis of prospective epidemiological studies. Sports Med 2014; 44(1):123-140. 